\title{
AVALIAÇÃO DA QUALIDADE FÍSICO-QUÍMICA DOS MEDICAMENTOS CONTENDO \\ DICLOFENACO DE POTÁSSIO
}

\author{
EVALUATION OF THE PHYSICAL-CHEMICAL QUALITY OF THE MEDICINES \\ CONTAINING DICLOFENAC POTASSIUM
}

\author{
FERNANDO CESAR QUEIROZ DA SILVA ${ }^{1}$; JESSICA VERTUAN RUFINO²; MARLENE \\ MARIA FREGONEZI NERY ${ }^{3}$
}

1 - Estagiário do Programa de Formação Complementar do Curso de Farmácia da Universidade Estadual de Londrina.

2 - Residente em Saúde da Mulher pela Universidade Estadual de Londrina.

3 - Docente da disciplina de Controle de Qualidade da Universidade Estadual de Londrina.

Autor para correspondência: fernandocqsilva@hotmail.com

\section{RESUMO:}

Os medicamentos anti-inflamatórios são umas das classes de medicamentos mais comercializadas no Brasil, sendo a subclasse dos anti-inflamatórios não esteroidais (AINE's) a de maior prevalência. Para que a população possa utiliza-los de maneira segura, é necessário um controle de qualidade adequado. O diclofenaco de potássio pertence ao grupo AINES's e é utilizado para tratamento em curto prazo de algumas condições agudas. O objetivo do trabalho foi avaliar a qualidade de medicamentos contendo diclofenaco de potássio $50 \mathrm{mg}$ de diferentes indústrias farmacêuticas. As amostras foram analisadas utilizando os parâmetros: peso médio, dureza, friabilidade, teor de princípio ativo, dissolução e uniformidade de dose unitária (UDU) de três especialidades farmacêuticas, sendo: três genéricos (G1, G2 e G3), um similar (S) e um referência (R). Todas as amostras foram aprovadas nos ensaios de friabilidade, apresentando para R, S, G1, G2 e G3, respectivamente $0,05 \%, 0,04 \%, 0,02 \%, 0,03 \%$ e $0,11 \%$. No ensaio de teor os valores para as amostras R, S, G1, G2 e G3, foram 104,29\% $(1,13), 96,12 \%(0,76), 99,11 \%(0,59)$, $98,09 \%(2,09), 101,96 \%(0,74)$. No ensaio de UDU obteve-se os valores de aceitação (VA) de 7,87, 4,27, 3,31, 2,31 e 6,07, respectivamente para R, S, G1, G2 e G3, portanto menor que 15. No teste de dissolução as amostras G1 e G3 não atenderam as especificações.

Palavras-chave: Diclofenaco de potássio, controle de qualidade.

\section{ABSTRACT:}

The anti-inflammatory drugs are one of the classes of medicines more marketed in Brazil, being the subclass of Nonsteroidal Anti-Inflammatory Drugs (NSAID's) with higher prevalence. So that the population can use it safely, proper quality control is required. Diclofenac potassium belongs to class of NSAID's and is used for short-term treatment of some acute conditions. The objective of the study was to evaluate the quality of medicines containing diclofenac potassium $50 \mathrm{mg}$ of differents pharmaceutical industries. The samples were analyzed using the parameters weight variation, hardness, friability, drug content, dissolution and dose uniformity (DU) of three pharmaceutical specialties, being: three generic drugs (G1, G2 and G3), one similar drug (S) and one reference drug (R). All samples were approved in the friability tests, presenting for R, S, G1, G2 and G3, respectively $0,05 \%$, $0,04 \%, 0,02 \%, 0,03 \%$ e $0,11 \%$. In the drug content test the values for the samples R, S, G1, G2 and G3, were 104,29\% (1,13), 96,12\% (0,76), 99,11\% (0,59), 98,09\% (2,09), 101,96\% $(0,74)$. In UDU teste was obtained acceptance value (AV) of 7,87, 4,27, 3,31, 2,31 and 6,07, 
respectively for $\mathrm{R}, \mathrm{S}, \mathrm{G} 1, \mathrm{G} 2$ and $\mathrm{G} 3$, therefore, less than 15 . In the dissolution test the samples $\mathrm{G} 1$ and $\mathrm{G} 3$ (were disapproved) does not meet the required specifications.

Key words: Potassium Diclofenac, quality control.

\section{INTRODUÇÃO}

A inflamação é uma resposta do organismo frente à lesão celular e, caracteriza-se por ser um fenômeno complexo promovido por diferentes agentes lesivos, como do tipo físico, biológico ou químico. A resposta a estas lesões caracteriza-se pelos sinais clínicos de rubor, calor, tumor e dor (NAGEL; SHIELDS, 2009; ISSY; SAKATA, 2008).

Os anti-inflamatórios não esteroides (AINES) estão entre os medicamentos mais comumente utilizados no mundo, estes exercem seus efeitos benéficos anti-inflamatório, analgésicos e antipiréticos pela inibição da cicloxigenase (COX), enzima chave na indução da síntese de prostaglandinas (GARNER,1992).

O diclofenaco de potássio (Figura 1), sal de potássio do ácido 2[(2,6diclorofenil)amino] benzenoacético, pertence ao subgrupo dos AINE's derivados do ácido fenilacético e é utilizado principalmente na forma de sal sódico ou potássico. Este fármaco é indicado para tratamento de curto prazo para as seguintes condições agudas: estado de dor inflamatória pós-traumática e pós-operatória; sintomas dolorosos da coluna vertebral; reumatismo não articular e no tratamento da dor, inflamação e febre que acompanham os processos infecciosos de ouvido, nariz e garganta. (PHYSICIANS' DESK REFERENCE, 2012).

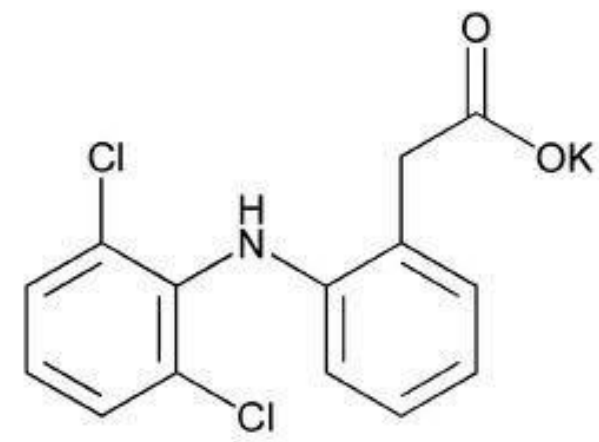

FIGURA 1. ESTRUTURA DO DICLOFENACO DE POTÁSSIO (BRASIL,2010a).

O diclofenaco de potássio é um pó cristalino branco, ligeiramente higroscópico; ligeiramente solúvel em água, facilmente solúvel em metanol e solúvel em etanol. Pode ser administrado por via oral, intramuscular, retal ou tópica. As apresentações farmacêuticas 
encontradas no mercado são comprimidos, pomadas, gel e supositório (GELLER. et al., 2012).

Com a diversidade de produtos oferecidos no mercado nacional, o controle de qualidade é essencial para garantir que um medicamento não trará prejuízos à saúde da população, pois se sabe que um mesmo fármaco produzido na mesma concentração e em uma mesma forma farmacêutica pode apresentar discrepâncias nas características físicoquímicas quando comparadas com marcas diferentes, devido a diversos fatores como a qualidade das matérias-primas e métodos de produção (PINTO; KANEKO; OHARA, 2000).

Segundo Ansel, Popovich e Allen-Jr (2000) muitos fatores podem comprometer a qualidade dos medicamentos, como, a utilização de matéria prima e material de embalagem de qualidade duvidosa, adoção de processos de fabricação inadequados, além de fatores que podem afetar a sua estabilidade (temperatura, armazenamento e transporte). Diante dos fatos, é muito importante avaliar a qualidade dos medicamentos fabricados por laboratórios farmacêuticos no Brasil, uma vez que estes produtos encontram-se disponíveis à população em farmácias, drogarias e Unidades Básicas de Saúde. O objetivo do presente trabalho foi avaliar a qualidade de comprimidos de diclofenaco de potássio (medicamento de referência, genérico e similar), provenientes de cinco laboratórios, utilizando a monografia do produto da Farmacopeia Brasileira 5ª Edição (BRASIL, 2010a).

\section{METODOLOGIA}

Foram analisados comprimidos de diclofenaco de potássio $50 \mathrm{mg}$ obtidos em Unidades Básicas de Saúde (UBS) e farmácias de Londrina, denominados G1, G2, G3 (Genéricos 1, 2 e 3), R (Referência) e S (Similar).

Todas as análises foram realizadas de acordo com o descrito na Farmacopeia Brasileira 5a edição (BRASIL, 2010a).

\subsection{Aspecto}

No teste de aspecto visual analisaram-se as amostras quanto à presença de deformações, rachaduras, uniformidade da coloração, tamanho e formato dos comprimidos, comprimidos faltantes, quebrados ou trincados, legibilidade na embalagem e qualquer outra alteração aparente.

\subsection{Peso Médio}


Para determinação do peso médio, pesou-se individualmente 20 comprimidos de cada amostra em balança analítica (Mettler, AE200) e calculou-se a média, os limites de variação e desvio padrão.

\subsection{Dureza e Friabilidade}

Os ensaios de dureza (MOD 298 ATTS) e friabilidade (MOD 300 Ethink) foram realizados, respectivamente, com 10 e 20 comprimidos. Para dureza, a força necessária para romper o comprimido foi dada em Newtons (N). Para a friabilidade, cada amostra foi submetida a 25 rotações por minuto (rpm) por quatro minutos (100 rotações) e calculada a perda percentual de massa das 20 unidades, baseada na diferença de valores do peso inicial e final.

\subsection{Dissolução}

Para o teste de dissolução, a princípio foram testados seis comprimidos de cada amostra no primeiro estágio (E1) em dissolutor (Erweka DT6), posteriormente, as amostras que não atenderam aos requisitos mínimos para aprovação no $\mathrm{E} 1$, foram analisadas mais seis comprimidos em um segundo estágio (E2). Para tal, utilizou-se: $900 \mathrm{~mL}$ de tampão fosfato de potássio $\mathrm{pH} 6,8$ em cada cuba, pás, $40 \mathrm{rpm}$, temperatura do meio $37^{\circ} \mathrm{C}\left( \pm 0,5^{\circ} \mathrm{C}\right)$ e tempo de coleta de 60 minutos. Após 60 minutos foram retiradas alíquotas de $10 \mathrm{~mL}$ do meio de dissolução de cada cuba, que após filtrada transferiu-se $3 \mathrm{~mL}$ do filtrado para balão volumétrico de $10 \mathrm{~mL}$, completou-se o volume com tampão fosfato de potássio $\mathrm{pH} \mathrm{6,8.} \mathrm{A}$ porcentagem dissolvida foi determinada em espectrofotômetro (Ethik- DTSA-60) a 276nm, após zerar o equipamento com tampão fosfato de potássio $\mathrm{pH} \mathrm{6,8.} \mathrm{Preparou-se} \mathrm{a} \mathrm{solução}$ padrão seguindo o mesmo procedimento.

\subsection{Doseamento}

Para o preparo das amostras, pesou-se do pó de 20 comprimidos triturados, o equivalente a $50 \mathrm{mg}$ de diclofenaco de potássio, em triplicata e transferiu-se quantitativamente para balão volumétrico de $100 \mathrm{~mL}$. Adicionou-se $70 \mathrm{~mL}$ de hidróxido de sódio 0,1 M, agitou-se em ultrassom por 15 minutos e completou-se o volume com o mesmo 
solvente. Esta solução foi filtrada e transferido $5 \mathrm{~mL}$ do filtrado em balão volumétrico de $50 \mathrm{~mL}$, completando o volume com $\mathrm{NaOH}$ 0,1 M; em seguida adicionou-se $10 \mathrm{~mL}$ da última solução em balão volumétrico de $25 \mathrm{~mL}$ e completou-se com o mesmo solvente.

Para o preparo da solução padrão, pesou-se exatamente $50 \mathrm{mg}$ de padrão secundário de diclofenaco de potássio, então, foi transferido para balão volumétrico de 100 $\mathrm{mL}$ e procedeu-se conforme o preparo da amostra. A concentração das soluções amostras e padrão foram determinadas em espectrofotômetro (Ethik - DTSA-60) a 276nm, após zerar o equipamento com hidróxido de sódio 0,1 M.

\subsection{Uniformidade de Dose Unitária}

O ensaio de uniformidade de dose unitária (UDU) foi realizado pelo método de variação de peso (VP), da seguinte forma: separaram-se 30 unidades de cada amostra e analisou-se, individualmente, 10 unidades. Pesou-se 10 unidades e estimou-se a quantidade do fármaco por unidade a partir do resultado do doseamento e dos pesos individuais, assumindo-se distribuição homogênea do componente ativo. As quantidades individuais estimadas (xi) foram calculadas segundo a equação $x i=\pi * A / P$, sendo, $\pi$ os pesos individuais das unidades ou dos conteúdos das unidades testadas; $A$ quantidade de componente ativo expressa em porcentagem da quantidade declarada, determinada no doseamento; e $P$ o peso médio das unidades utilizadas no doseamento. Em seguida, foram calculados os valores de aceitação (VA) pela equação $V A=|M-\bar{X}|+k s$ onde, $X$ é a média dos conteúdos individuais, expressa como porcentagem da quantidade declarada; Mo valor de referência; $K$ a constante de aceitabilidade $(k=2,4$ para 10 unidades e 2,0 para 30 unidades); e s o desvio padrão da amostra (BRASIL, 2010a).

\section{RESULTADOS E DISCUSSÃO}

Segundo a Farmacopeia Brasileira 5a edição (BRASIL, 2010a) a variação de peso aceitável para comprimidos, com peso médio entre 80 mg a 250 mg é de 7,5\% (S e G3) e, para comprimidos com peso superior a $250 \mathrm{mg}$ é de $\pm 5 \%$ (R, G1 e G2), não sendo permitido mais do que duas unidades fora destes limites. A Tabela 1 apresenta os resultados de peso médio e dureza para cada amostra analisada. Em relação ao peso médio, todas as amostras cumpriram os requisitos estabelecidos, demonstrando que o processo de 
fabricação, no quesito peso médio encontra-se adequado, sugerindo que houve correto preenchimento da matriz e regulagem das punções superior e inferior (MOISÉS, 2006).

TABELA 1. VALORES OBTIDOS NA DETERMINAÇÃO DE PESO MÉDIO E DUREZA DE COMPRIMIDOS DE DICLOFENACO DE POTÁSSIO 50MG.

\begin{tabular}{|c|c|c|}
\hline Amostras & Peso médio (mg) & Dureza (N) \\
\hline $\mathbf{R}$ & $321,97(305,87-338,07)^{\star}(8,82)^{* \star}$ & $65,20(14,09)^{* *}$ \\
\hline $\mathbf{S}$ & $183,97(174,77-192,17)^{\star}(2,64)^{\star *}$ & $153,3(10,60)^{\star *}$ \\
\hline G1 & $260,95(247,90-273,99)^{*}(4,63)^{\star *}$ & $84,40(13,30)^{\star *}$ \\
\hline G2 & $267,05(253,69-280,40)^{\star}(2,26)^{\star \star}$ & $125,90(6,70)^{* *}$ \\
\hline G3 & $189,08(179,63-198,53)^{*}(3,51)^{\star *}$ & $72,20(10,00)^{* *}$ \\
\hline
\end{tabular}

A dureza é uma característica física muito importante para comprimidos, pois garante a integridade desta forma farmacêutica a choques mecânicos durante os processos de revestimento, embalagem e transporte. Sua determinação está relacionada com a desintegração e consequentemente com a dissolução do princípio ativo (FERRAZ et al., 2000). Como apresentado na TABELA 1, as amostras R, S, G1 e G3 apresentaram valores elevados ao desvio padrão do parâmetro dureza, indicando provavelmente a ocorrência de não calibração da força exercida pelas punções da máquina compressora, por isso julgase necessário à exigência de programas de calibração e qualificação periódicos destes equipamentos (BRASIL, 2010b). Ainda assim, pode-se considerar estes resultados aceitáveis já que a literatura oficial não traz padrões máximos para este teste.

No parâmetro friabilidade, todas as amostras foram aprovadas seguindo os requisitos da Farmacopeia Brasileira $5^{\underline{a}}$ ed., sendo que os valores das amostras $\mathrm{R}, \mathrm{S}, \mathrm{G} 1$, G2 e G3 apresentaram, respectivamente, 0,05\%, 0,04\%, 0,02\%, 0,03\% e 0,11\%.

$\mathrm{O}$ teste de doseamento visa quantificar o teor de princípio ativo contido nos comprimidos analisados, podendo ter uma margem na variação de 90,00\% a 110,00\% no caso do diclofenaco de potássio (BRASIL, 2010a). Analisando os dados da Tabela 2, observa-se que todas as amostras analisadas (R, S, G1, G2 e G3) cumpriram o requisito para este teste. 
TABELA 2. RESULTADOS OBTIDOS NO TESTE DE DOSEAMENTO EM COMPRIMIDOS CONTENDO DICLOFENACO DE POTÁSSIO 5OMG POR ESPECTROFOTOMETRIA NO UV.

\begin{tabular}{cc}
\hline Amostra & Teor \% (DP) \\
\hline R & $104,29(1,13)$ \\
S & $96,12(0,76)$ \\
G2 & $99,11(0,59)$ \\
G3 & $98,09(2,09)$ \\
\hline
\end{tabular}

R (Referência), G1, 2 e 3 (Genérico 1, 2 e 3), S (Similar), DP (Desvio Padrão)

O ensaio de dissolução determina a porcentagem do fármaco que é liberado no meio de dissolução nas condições definidas (BRASIL, 2010). A dissolução está relacionada com a biodisponibilidade do fármaco no organismo, para tanto, é imprescindível que estes sejam dissolvidos, liberando uma porcentagem do princípio ativo para o meio de dissolução, a fim de que o mesmo possa exercer a sua ação farmacológica (PEIXOTO et al., 2005). A Tabela 3 mostra os resultados obtidos no ensaio de dissolução para todas as amostras.

TABELA 3. VALORES DE PORCENTAGEM DISSOLVIDA DE DICLOFENACO DE POTÁSSIO 50MG, EM COMPRIMIDOS, COM MEIO DE DISSOLUÇÃO TAMPÃO FOSFATO pH 6,8 $\left(37 \pm 0,5^{\circ} \mathrm{C}\right)$, PÁ, 40 RPM EM 60 MIN.

\begin{tabular}{ccccccc}
\hline Cubas & $\mathbf{1}$ & $\mathbf{2}$ & $\mathbf{3}$ & $\mathbf{4}$ & $\mathbf{5}$ & $\mathbf{6}$ \\
\hline $\mathbf{R}^{\star}$ & $\mathbf{7 1 , 0 0}$ & 95,31 & 97,58 & 83,00 & 92,18 & 97,58 \\
$\mathbf{R}^{\star *}$ & 99,59 & 99,17 & 85,08 & 94,48 & 94,89 & 95,86 \\
$\mathbf{S}^{*}$ & 95,10 & 97,06 & 93,92 & 94,06 & 94,71 & 96,67 \\
G1 $^{*}$ & 87,86 & 87,40 & 91,40 & $\mathbf{3 9 , 0 0}$ & 121,00 & $\mathbf{6 9 , 0 0}$ \\
G2 $^{*}$ & 85,56 & 85,10 & 90,94 & $\mathbf{8 3 , 1 0}$ & $\mathbf{7 7 , 1 2}$ & $\mathbf{7 3 , 4 3}$ \\
G2 $^{* *}$ & 88,00 & 90,63 & 108,00 & $\mathbf{7 7 , 2 7}$ & $\mathbf{7 4 , 5 0}$ & $\mathbf{8 1 , 4 1}$ \\
G3 $^{*}$ & $\mathbf{3 9 , 1 9}$ & $\mathbf{5 5 , 4 1}$ & $\mathbf{4 9 , 6 1}$ & $\mathbf{4 1 , 1 2}$ & $\mathbf{5 6 , 3 7}$ & $\mathbf{5 5 , 9 8}$ \\
\hline
\end{tabular}

R (Referência), G1, 2 e 3 (Genérico 1, 2 e 3), S (Similar); Ensaio $1\left(^{*}\right)$; Ensaio $2\left(^{* *}\right)$

O método estabelecido pela farmacopeia rege que a porcentagem mínima de princípio ativo liberado de cada comprimido seja de $80 \%(Q+5 \%)$ no primeiro estágio (E1). 
Conforme apresentado na Tabela 6, a única a amostra que cumpriu os requisitos estabelecidos no $\mathrm{E} 1$ foi a amostra $\mathrm{S}$. As amostras $\mathrm{R}$ e $\mathrm{G} 2$ não cumpriram os requisitos no E1, apresentado nas cubas $1(73 \%)$ e $4(83 \%)$ para R e cubas $4(77,27 \%), 5(74,50 \%)$ e 6 $(81,41 \%)$ para G2. Ambas as amostras passaram para o segundo estágio (E2), sendo então analisadas mais seis unidades de cada e, neste caso, a média das doze unidades analisadas não poderia ser menor do que $80 \%$, e nenhuma unidade menor que $65 \%$ (Q15\%). As amostras R e G2 foram aprovadas no E2 com médias respectivas de 92,14\% e $84,59 \%$ e nenhuma unidade foi menor que $65 \%$. Já, as amostras G1 e G3 não cumpriram os requisitos estabelecidos pela Farmacopeia, sendo então reprovadas já no E1 por apresentarem unidades com porcentagem liberada abaixo de $50 \%$, cuba $4(39,0 \%)$ para G1 e cuba $1(39,19 \%), 3(49,61 \%)$ e $4(41,12 \%)$ para G3.

Os resultados do ensaio de uniformidade de dose unitárias calculados por meio do VA para as amostras R, S, G1, G2 e G3 foram, respectivamente de, 7,87, 4,27, 3,31, 2,31 e 6,07, portanto estes resultados significam que os comprimidos testados apresentam uma distribuição homogênea da substância ativa por unidade, o que garante a dose correta no tratamento.

Lotes de medicamentos contendo diclofenaco de potássio são comumente recolhidos pela ANVISA por não estarem em conformidade. Um levantamento realizado no site de produtos irregulares da ANVISA mostrou que foram publicadas nove Resoluções específicas no período de 2010 a 2016, exigindo o recolhimento do mercado de diferentes lotes de medicamentos que continham diclofenaco. Isto mostra a necessidade e importância de serem seguidas as boas práticas de fabricação (BRASIL, 2010b), para que sejam cumpridos os requisitos estabelecidos pela Farmacopeia Brasileira (BRASIL, 2010a).

\section{CONCLUSÃO}

A avaliação da qualidade dos medicamentos pela Indústria Farmacêutica antes de sua comercialização é de grande importância, visto a quantidade de notificações e recolhimentos publicados pela ANVISA. O controle de qualidade faz uso de um conjunto de medidas destinadas a verificar a qualidade de cada lote de medicamentos, para certificar se estes satisfazem o tripé, qualidade, eficácia e segurança. Tendo em vista os resultados obtidos pelas análises feitas, as amostras G1 e G3 não estariam aptas para comercialização, visto a reprovação de ambas no teste de dissolução. Esta não conformidade pode ter ocorrido por diversos fatores, desde a composição do medicamento, 
até o não cumprimento das Boas Práticas de Fabricação, podendo causar ineficácia terapêutica e riscos a saúde do paciente.

\section{REFERÊNCIAS}

ANSEL, H. C.; POPOVICH, N. G., ALLEN-JR. L. V. Farmacotécnica: formas farmacêuticas e sistemas de liberação de fármacos. 6ª ed. São Paulo: Premier, 2000. p.716.

BRASIL. Ministério da Saúde. Agencia Nacional de Vigilância Sanitária. Farmacopeia Brasileira: Volume 2 - Monografias. 5ª ed. Brasília, 2010. p. 906-907.a

BRASIL. Ministério da Saúde. Agência Nacional de Vigilância Sanitária. Resolução da Diretoria Colegiada (RDC) no 17, de 16 de abril de 2010. Disponível em: <http://portal.anvisa.gov.br/documents/33880/2568070/res0017_16_04_2010.pdf/b9a8a29 3-f04c-45d1-ad4c-19e3e8bee9fa>. Acesso em: 10 out. 2017.b

FERRAZ, G. H. et al. Avaliação do perfil de dissolução de especialidades farmacêuticas contendo diclofenaco sódico sob a forma de comprimidos de liberação entérica. Revista Ciênc. Farm. v. 21, n. 2, p. 191-199, 2000.

GARNER, A.. Adaptation in the Pharmaceutical Industry, with Particular Reference to Gastrointestinal Drugs and Diseases. Scandinavian Journal Of Gastroenterology, [s.I.], v. 27, n. 193, p.83-89, jan. 1992.

GELLER, M. et al. Utilização do diclofenaco na prática clínica: revisão das evidências terapêuticas e ações farmacológicas. Revista Brasileira de Clínica Medica, v. 10, n. 1, p. 29-38, 2012.

ISSY, A. M.; SAKATA R. K. Fármacos para o tratamento da dor. São Paulo: Manole, 2008. p.325.

MOISÉS, P. R. Tecnologia de Produção de Comprimidos. Fármacos e Medicamentos, São Paulo, v. 7, n.38, p.38-46, janeiro de 2006. 
NAGEL D. S.; SHIELDS H. M. Farmacologia integrativa da inflamação: doença ulcerosa péptica. In: Golan DE, Tashjian - Jr AH, Armstrong EJ, Armstrong AW. Princípios de Farmacologia. Rio de Janeiro: Guanabara Koogan, 2009. cap. 45, p. 759-763.

PINTO, T. J. A.; KANEKO, T. M.; OHARA, M. T. Controle Biológico de Qualidade de Produtos Farmacêuticos, Correlatos e Cosméticos. $2^{\mathrm{a}}$ ed. São Paulo: Atheneu, 2000. p.325.

PEIXOTO, Maíra Moreira et al. AVALIAÇÃO DA QUALIDADE DE COMPRIMIDOS DE CAPTOPRIL DISPENSADOS EM FEIRA DE SANTANA - BA. Infarma, v. 16, n. 13-14, p.69-73, 2005

Physicians' Desk Reference. 66 ed. Montvale: Medical Economics Company, New Jersey, 2012. 